\title{
THE
}

$2-11-1980$

\section{Flow Behavior of an Oriented, Reentrant, Nematic Liquid Crystal}

S. Bhattacharya

S. V. Letcher

University of Rhode Island, sletcher@uri.edu

Follow this and additional works at: https://digitalcommons.uri.edu/phys_facpubs

Terms of Use

All rights reserved under copyright.

\section{Citation/Publisher Attribution}

Bhattacharya, S., \& Letcher, S. V. (1980). Flow Behavior of an Oriented, Reentrant, Nematic Liquid Crystal. Physical Review Letters, 44(6), 414-417.

Available at: http://dx.doi.org/10.1103/PhysRevLett.44.414

This Article is brought to you for free and open access by the Physics at DigitalCommons@URI. It has been accepted for inclusion in Physics Faculty Publications by an authorized administrator of DigitalCommons@URI. For more information, please contact digitalcommons-group@uri.edu. 


\title{
Flow Behavior of an Oriented, Reentrant, Nematic Liquid Crystal
}

\author{
S. Bhattacharya ${ }^{(a)}$ and S. V. Letcher \\ Department of Physics, University of Rhode Island, Kingston, Rhode Island 02881, and \\ Francis Bitter National Magnet Laboratory, Massachusettes Institute of Technology, \\ Cambridge, Massachusettes 02139 \\ (Received 5 September 1979)
}

\begin{abstract}
Capillary shear flow of a magnetically aligned liquid crystal was studied in the three Miesowicz orientations in the nematic, the smectic- $A$ and, for the first time, in the reentrant nematic phase. These results indicate that the flow behavior of the reentrant nematic phase is essentially identical to that of the nematic phase and can be described by the hydrodynamic theory of the nematic phase.
\end{abstract}

An unusual phase diagram of certain liquid crystal mixtures was reported originally by Cladis. ${ }^{1}$ Upon lowering the temperature, certain concentrations undergo successive phase transitions; isotropic $(I)$, nematic $(N)$, smectic- $A$, and nematic again. The lower-temperature phase is now called the reentrant nematic $\left(N^{*}\right)$ phase. The same sequence has been observed in single-component systems on the application of pressure. ${ }^{2}$ A model for the molecular arrangement has been proposed $^{3}$ and a Landau theory based on the assumption of the existence of an optimum density (or concentration in a mixture) for smectic ordering has also been formulated. ${ }^{4}$ Little is known, however, about the dynamic properties of this phase; specifically it has not been demonstrated that the hydrodynamic theories of nematics adequately describe its behavior.

The bulk of the experimental studies have been performed on systems where the reentrant nematic phase is in a supercooled state. Recent $x$-ray scattering studies, ${ }^{5}$ for example, showed that the reentrant nematic phase coexists with microcrystallites, both melting to the smectic- $A$ phase at the same temperature. This led these authors to conclude that (i) the reentrant nematic is different from the high-temperature nematic and (ii) the reentrant nematic may share some of the struc- tural properties of the crystalline phase tablish the nature of long-range symmetries of the reentrant nematic phase it is important to study stable systems.

In this Letter we present the first experimental evidence, from capillary flow measurements in well-defined geometries, that the flow behavior of a stable reentrant nematic phase is essentially identical to that of the ordinary nematic and qualitatively distinct from that of the intermediate smectic- $A$ phase. We demonstrate that the longrange orientational order persists in the reentrant nematic phase and the continuous translational symmetry along the director is restored。 The hydrodynamics of the nematic phase then describes the stable reentrant nematic。

The material chosen for study was a mixture of heptylcyanobiphenyl (7CB), octyloxycyanobiphenyl (80CB), and pentylcyanoterphenyl (5CT) in the ratio of $73.18: 9$ by weight. ${ }^{6}$ The mixture has the following transition temperatures:

$$
N * \stackrel{10.1{ }^{\circ} \mathrm{C}}{\longrightarrow} A \stackrel{26.9^{\circ} \mathrm{C}}{\longleftrightarrow} N \stackrel{65.6{ }^{\circ} \mathrm{C}}{\longleftrightarrow} I .
$$

In addition to good chemical stability, this material has the two crucial advantages that the reentrant nematic $\left(N^{*}\right)$ phase is stable, not supercooled, and secondly, the transitions are com-

TABLE I. Miesowicz geometries and effective viscosities.

\begin{tabular}{ccccc}
\hline Orientation & Geometry & $\begin{array}{c}\text { Miesowicz } \\
\text { viscosity }\end{array}$ & $\begin{array}{c}\text { Effective viscosity } \\
\text { Leslie notation }{ }^{2}\end{array}$ FLMSP notation ${ }^{b}$ \\
\hline (a) & $\vec{v} \perp \hat{n}$ & $\eta_{a}$ & $\frac{1}{2} \alpha_{4}$ & $\nu_{2}$ \\
& $\nabla v \perp \hat{n}$ & & & \\
(b) & $\overrightarrow{\mathrm{v}} \| \hat{n}$ & $\eta_{b}$ & $\frac{1}{2}\left(\alpha_{3}+\alpha_{4}+\alpha_{6}\right)$ & $\nu_{3}+\gamma_{1} / 4(1-\lambda)^{2}$ \\
& $\nabla v \perp \hat{n}$ & & & \\
(c) & $\overrightarrow{\mathrm{v}} \perp \hat{n}$ & $\eta_{c}$ & $\frac{1}{2}\left(\alpha_{4}+\alpha_{5}-\alpha_{2}\right)$ & $\nu_{3}+\gamma_{1} / 4(1+\lambda)^{3}$ \\
\hline
\end{tabular}

${ }^{\mathrm{a}}$ F. M. Leslie, Arch. Ration. Mech. Anal. 28 , 265 (1968).

${ }^{b}$ Ref. 7 . 


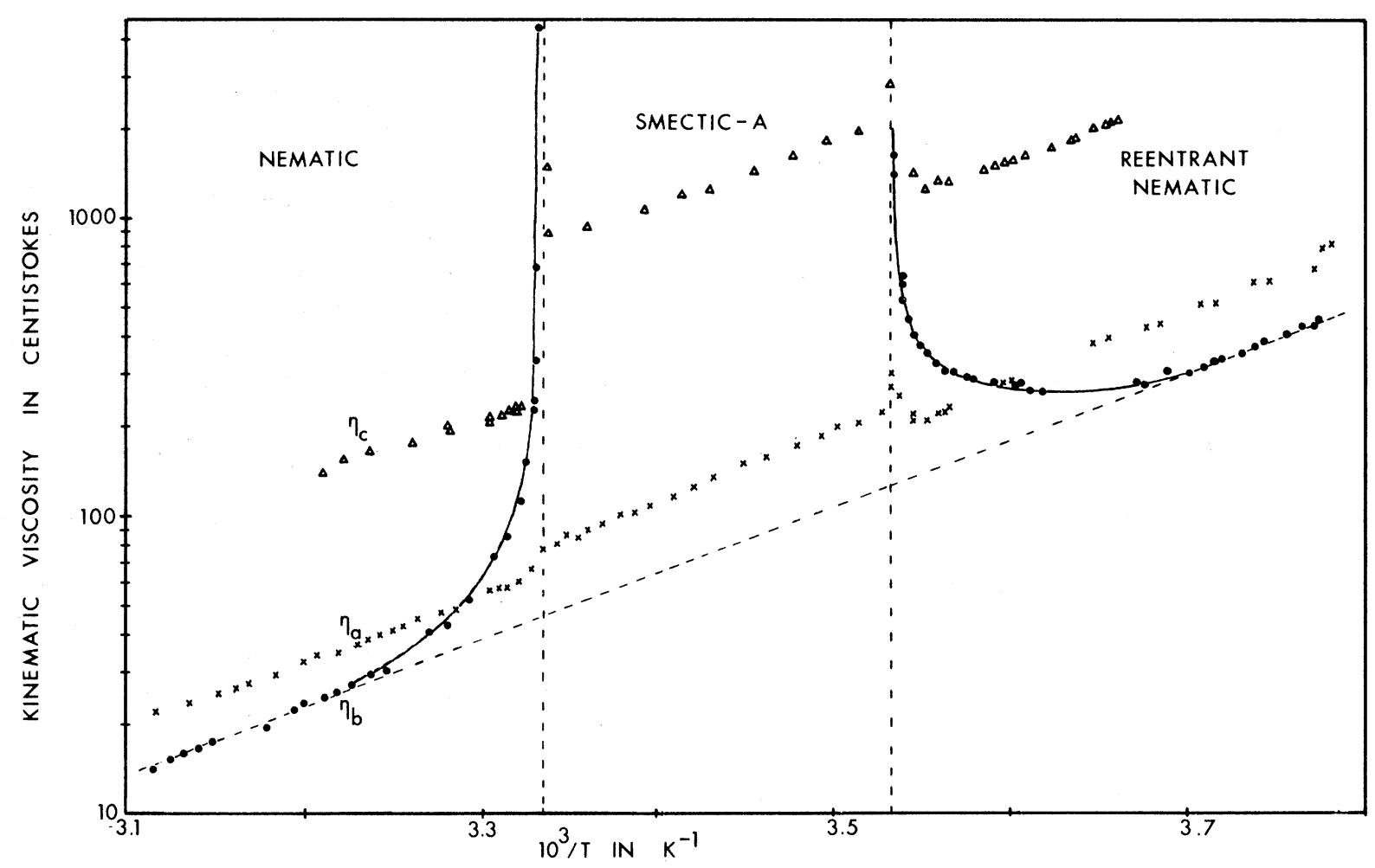

FIG. 1. Temperature dependence of the three Miesowicz viscosities in the nematic, smectic- $A$, and the reentrant nematic phases. Crosses, orientation $(a)$; solid circles, orientation $(b)$; triangles, orientation $(c)$. The dashed line is the Arrhenius background for $\eta_{b}$. The solid curves are guides for the eye.

pletely reversible. The transitions are moreover, almost second order ${ }^{6}$; the McMillian number, $T_{N A} / T_{N I}$, is $\sim 0.88$.

Capillary shear flow was studied in the three Miesowicz orientations, summarized in Table I. Orientations $(a)$ and $(c)$ were studied in surfacetreated flat capillaries with rectangular cross section (thickness $\sim 150 \mu \mathrm{m}$, width $\sim 1 \mathrm{~cm}$, length $\sim 5 \mathrm{~cm}$ ) while orientation $(b)$ was studied in a capillary of circular cross section ( 1 mm i.d.). The details of the viscometers and the measurement techniques are given elsewhere. ${ }^{8}$ The alignment in the bulk was obtained by application of a magnetic field $(H \sim 70 \mathrm{kOe})$, which prevented flow alignment and the consequent non-Newtonian effects. The viscometers were calibrated against a calibrated Ubbelhode viscometer. The temperature was controlled to within $\pm 0.1 \mathrm{~K}$ by a circulating methanol bath.

The temperature dependences of the kinematic viscosities in the three orientations are shown in Fig. 1. We make the following observations:

(i) The flow in all geometries is Newtonian in the $N$ and $N^{*}$ phases. Non-Newtonian effects are present in the $(a)$ orientation of the smectic- $A$ phase. Typically a $100 \%$ increase in the average shear stress causes a 15-20\% decrease in the apparent viscosity. The data for the $(a)$ orientation of the $A$ phase in Fig. 1 represent the effective viscosities at shear rates comparable to the ones used in the $N$ and $N^{*}$ phases. The apparent viscosities in the $A$ phase are consistently higher than the extrapolated values in the $N$ and $N^{*}$ phases; they would be even higher at lowershear rates. The non-Newtonian behavior of this orientation of smectic $A$ seems to be characteristic of capillary-flow measurements. ${ }^{8}$

(ii) The temperature dependences of the viscosities $\eta_{a}$ and $\eta_{c}$ are roughly Arrhenius in both $N$ and $N^{*}$ phases with approximately the same activation energies, $E_{a} \sim 10-11 \mathrm{kcal} / \mathrm{mole}$ and $E_{c}$ $\sim 8-10 \mathrm{kcal} / \mathrm{mole}$.

(iii) The apparent viscosity for the $(c)$ orientation shows a shear-rate-dependent peak on entering the $A$ phase from above and from below. A similar effect is seen at the $N^{*}-A$ transition in the $(a)$ orientation. This is qualitatively similar to the flow-alignment-induced non-Newtonian peak observed at the isotropic-cholesteric transition in unoriented samples, ${ }^{9}$ but has not been seen be- 
fore in this type of experiment. The origin of this effect is not clear.

(iv) The viscosity in geometry (b) shows a striking divergence as the $A$ phase is approached from above and from below. The flow is blocked in the $A$ phase. Far away from the transitions, the viscosity is roughly Arrhenius in both $N$ and $N^{*}$ phases with activation energy $E_{b} \sim 11 \mathrm{kcal} / \mathrm{mole}$.

The flow behavior in the $N$ phase and near the $N-A$ transition is consistent with the results in other nematics and other $N-A$ transitions. ${ }^{8,10}$

Far away from the transition in the nematic phase we find the expected anisotropy in the viscosity: $\eta_{c}>\eta_{a}>\eta_{b}$. Theories of the dynamics of the $N-A$ phase transition predict ${ }^{11}$ that the FLMSP coefficients ${ }^{7} \nu_{2}$ and $\nu_{3}$ are regular at the transition, $\gamma_{1}$ diverges, and $\lambda$ takes on an asymptotic value of - 1. The latter is responsible for $\eta_{c}$ remaining regular at the transition and for the divergence of $\eta_{b}$ at the transition being faster than that of $\gamma_{1}$ alone. From our measurements, one cannot determine the nature of the divergence of $\gamma_{1}$ alone. With the Arrhenius background (dashed line in Fig. 1) subtracted, the apparent exponent for $\eta_{b}$ is found to be $1.05 \pm 0.1$. This is larger than the predicted value ${ }^{11}$ for $\gamma_{1}$ and is consistent with the results at the $N-A$ transition of $N-p$-cyanobenzylidene-p-octyloxyaniline.

Returning to the reentrant nematic phase and the $N^{*}-A$ phase transition, the main interest of the present study, we note the following: (i) Far away from the transition, the viscosities return to the same anisotropy as in the nematic phase; i.e., $\eta_{c}>\eta_{a}>\eta_{b}$. (ii) For geometries (a) and (c) the rise in the viscosity on entering the $A$ phase is similar to that at the $N-A$ transition. (iii) Most importantly, $\eta_{b}$ diverges as the $A$ phase is approached from below in a way that is qualitatively identical to the $N-A$ transition.

The Martin-Parodi-Pershan (MPP) formalism ${ }^{12}$ of the liquid-crystal hydodynamics asserts that the modification of hydrodynamics for the smectic- $A$ phase over that of the nematic phase is caused by the appearance of a new hydrodynamic variable (displacement along the layer normal) corresponding to the broken uniform translational symmetry along that direction. This is responsible for the blocked flow for geometry $(b)$ at the onset of the permeation dominated regime. The fact that the flow we observe is no longer blocked in the reentrant phase and, furthermore, is Newtonian, signifies clearly the recovery of the uniform translational symmetry parallel to the director. This, therefore, implies that the origi- nal conservation relations of the nematic phase are restored in the reentrant nematic phase. Clearly, the same hydrodynamic theory should be valid in both phases.

In two ways the data in Fig. 1 are not symmetric about the $A$ phase. In geometry $(c)$, the Arrhenius plot gives the same activation energies in both nematic phases, but the intercepts are different. Even though the hydrodynamic theory describes the qualitative behavior of the reentrant phase, this quantitative asymmetry could have its roots in the difference in the short-range scale of the molecular arrangements of $N$ and $N^{*}$ phases. ${ }^{14}$ A free-volume approach to the viscosities could be useful in understanding this difference.

Also, the divergence of $\eta_{b}$ is not quantitatively symmetric. The exponent found after subtracting the Arrhenius background from the data at the $N^{*}-A$ transition is $0.59 \pm 0.1$, considerably less than at the $N-A$ transition. Whether this is a real difference or is due to incorrect background subtraction $^{15}$ is not known. Direct measurements of $\gamma_{1}$ and the nematic order parameter that should clarify the situation are in progress.

In conclusion, we have demonstrated that the orientational order persists in the reentrant nematic phase; the continuous translational symmetry along the director is also restored. The quantitative asymmetry about the smectic- $A$ phase may have its roots in the difference in the shortrange order. From the simple flow behavior of this stable reentrant nematic it appears that further studies of such stable systems would be useful in understanding the nature of this unusual phase.

We thank P. E. Cladis for helpful discussions and for bringing Ref. 6 to our attention. We also thank P. H. Keyes for the loan of equipment. We acknowledge technical assistance of P. Dain, W. Hamidzada, L. Lu, and J. Stroscio. This work was supported by the National Science Foundation under Grant No. DMR 77-07813.

\footnotetext{
(a) Present address: James Franck Institute, University of Chicago, Chicago, Ml. 60637.

${ }^{1}$ P. E. Cladis, Phys. Rev. Lett. 35, 48 (1975).

${ }^{2}$ P. E. Cladis, R. K. Bogardus, $\overrightarrow{\mathrm{W}}$. B. Daniels, and G. N. Taylor, Phys. Rev. Lett. 39, 720 (1977).

${ }^{3}$ P. E. Cladis, R. K. Bogardus, and D. Aadsen, Phys. Rev. A 18, 2292 (1978).

${ }^{4}$ P. S. Pershan and J. Prost, J. Phys. (Paris), Lett. 40, L-27 (1979).
} 
${ }^{5}$ D. Guillon, P. E. Cladis, and J. Stamatoff, Phys. Rev. Lett. $\underline{41}, 1598$ (1978).

${ }^{6}$ R. J. Holden and E. P. Raynes, in Proceedings of the Seventh International Liquid Crystals Conference, Bordeaux, France, 1978 (to be published).

${ }^{7}$ The notation was given by D. Forster, T. C. Lubensky, P. Martin, J. Swift, and P. Pershan, Phys. Rev. Lett. 26, 1016 (1971).

${ }^{8}$ M. G. Kim, S. Park, Sr. M. Cooper, and S. V. Letcher, Mol. Cryst. Liq. Cryst. 36, 143 (1976).

${ }^{9}$ T. Yamada and E. Fukuda, Jpn. J. Appl. Phys. 12, 68 (1973).

${ }^{10} \mathrm{Ch}$. Gähwiller, Mol. Cryst. Liq. Cryst. $\underline{20}, 301$
(1973) .

${ }^{11}$ See, for example, K. Hossain, J. Swift, J. H. Chen, and T. C. Lubensky, Phys. Rev. B 19, 432 (1979), and references therein.

${ }^{12}$ P. C. Martin, O. Parodi, and P. S. Pershan, Phys. Rev. A 6, 2401 (1973).

${ }^{13}$ W. Helfrich, Phys. Rev. Lett. 23,372 (1969).

${ }^{14}$ Analogies can be drawn to the liquid-vapor transition far away from the critical point where, in spite of the same long-range symmetry properties of the two phases, quantitative differences between the two phases exist.

${ }^{15}$ A. F. Martins, A. C. Diogo, and N. P. Vaz, Ann. Phys.(Paris) 3 , 361 (1978).

\title{
Stimulated Emission of $29-\mathrm{cm}^{-1}$ Phonons in Ruby
}

\section{P. $\mathrm{Hu}$}

Bell Laboratories, Murray Hill, New Jersey 07974

(Received 23 October 1979)

\begin{abstract}
With resonance $R_{2}$ laser light pumping, the observation of stimulated emission of the $29-\mathrm{cm}^{-1}$ phonon in ruby is reported. The transverse phonons propagate along the $C_{3}$ axis and have some nonlinear gain.
\end{abstract}

Recently, extensive works have been reported concerning optical generation or detection of single-frequency high-energy phonons. ${ }^{1-4}$ Typically, the generation of these high-frequency phonons was accomplished either by optical excitation through some broad absorption band or the standard technique of a thin heater film. The detector, which is at another part of the same sample, is usually a region where an appreciable amount of ions are maintained at some excited state. In the case of ruby, it is the $\bar{E}\left({ }^{2} E\right)$ as shown in Fig. 1. The detection of the single-frequency $29-\mathrm{cm}^{-1}$ phonons is then achieved by the observation of time-delayed $R_{2}$ light pulses. The delay time corresponds to the time of flight for phonons to travel ballistically from the generator to the detector. More recently, evidence of highenergy stimulated phonon emission excited by resonant infrared pumping in $\mathrm{Al}_{2} \mathrm{O}_{3}$ doped with $\mathrm{V}^{4+}$ was reported. ${ }^{5}$ I report here the observation of stimulated $29-\mathrm{cm}^{-1}$ phonon emission in ruby with resonant optical pumping based on the frequency and directionality of the phonon observed. Some nonlinear gain is also reported.

A Molectron UV $-400 \mathrm{~N}_{2}$ laser was used to pump a homemade dye laser with an intracavity prism beam expander. The 5-ns laser pulse had a peak power $\sim 10 \mathrm{~kW}$. The linewidth was $0.2 \mathrm{~cm}^{-1}$ which was somewhat larger than that of the $R_{2}$ line in our ruby samples. The dye laser was then fo-
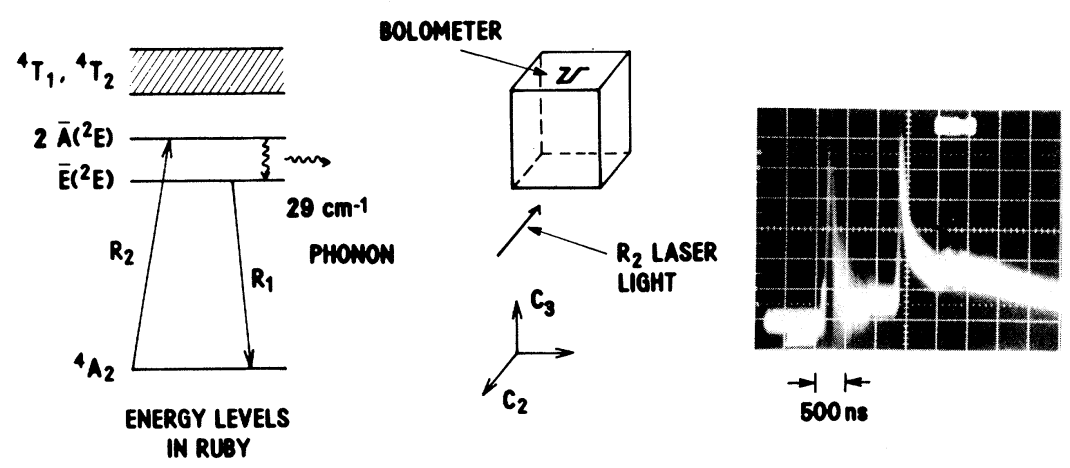

FIG. 1. Schematic energy level diagram in ruby and geometry of the resonance optical excitation experiment. The photograph shows the signals as observed on the scope with a granular aluminum bolometer. The initial and the time-delayed pulses are due to scattered laser light and transverse phonons, respectively. 